Supplement of Hydrol. Earth Syst. Sci., 25, 375-386, 2021

https://doi.org/10.5194/hess-25-375-2021-supplement

(c) Author(s) 2021. This work is distributed under

the Creative Commons Attribution 4.0 License.

(c) (1)

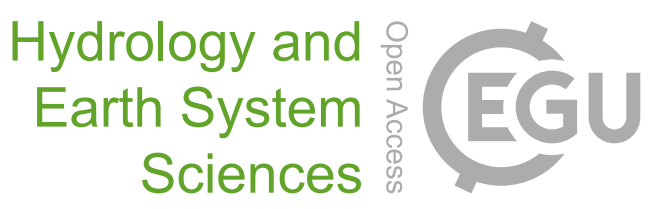

Supplement of

\title{
At which timescale does the complementary principle perform best in evaporation estimation?
}

\section{Liming Wang et al.}

Correspondence to: Fuqiang Tian (tianfq@ mail.tsinghua.edu.cn)

The copyright of individual parts of the supplement might differ from the CC BY 4.0 License. 


\section{Caption}

The information including IGBP (International Geosphere-Biosphere Programme)

types, location, study periods, measurement heights, canopy heights and energy

balance condition at the 88 eddy flux sites is listed in Table S1. Table S2 provides the

evaluation merits (NSE, $\mathrm{R}^{2}$ and RMSE in $\mathrm{W} \mathrm{m}^{-2}$ ) of the two generalized

complementary functions for each ecosystem types. Table S2 provides the evaluation merits for using the "Bowen ratio" (BR) closure correction method. Figure S1 gives relationship between the estimated evaporation and the observed site mean evaporation at seasonal scale. 
Table S1. Summary of the information at the 88 eddy flux sites.

\begin{tabular}{|c|c|c|c|c|c|c|c|c|}
\hline $\begin{array}{c}\text { Site } \\
\text { name }\end{array}$ & IGBP & LAT & LONG & Year & $\begin{array}{c}\text { Measurement } \\
\text { height (m) }\end{array}$ & $\begin{array}{c}\text { Canopy } \\
\text { height }(\mathrm{m})\end{array}$ & $\begin{array}{l}\text { Energy } \\
\text { balance } \\
\text { residual } \\
\left(\mathrm{W} \mathrm{m}^{-2}\right)\end{array}$ & $\begin{array}{c}\text { Energy } \\
\text { balance } \\
\text { closure } \\
\text { ratio } \\
\end{array}$ \\
\hline AT-Neu & GRA & 47.117 & 11.318 & 2002 2012 & 2 & 0.76 & 16.38 & 0.83 \\
\hline $\begin{array}{l}\text { AU- } \\
\text { ASM }\end{array}$ & ENF & -22.283 & 133.249 & 2010 2014 & 11.7 & 6.5 & 28.99 & 0.82 \\
\hline AU-Cpr & SAV & -34.002 & 140.589 & 2010 2014 & 20 & 5 & 17.36 & 0.88 \\
\hline AU-DaS & SAV & -14.159 & 131.388 & 2008 2014 & 20.4 & 16.4 & 25.21 & 0.84 \\
\hline AU-Dry & SAV & -15.259 & 132.371 & 2009 2014 & 16.3 & 12.3 & 31.20 & 0.80 \\
\hline AU-How & WSA & -12.494 & 131.152 & 2003 2014 & 23 & 15 & 17.43 & 0.89 \\
\hline AU-Stp & GRA & -17.151 & 133.350 & 2008 2014 & 4.2 & 0.2 & 5.15 & 0.96 \\
\hline AU-Tum & EBF & -35.657 & 148.152 & 2001 2014 & 70 & 40 & 21.79 & 0.88 \\
\hline $\begin{array}{l}\text { AU- } \\
\text { Wom }\end{array}$ & $\mathrm{EBF}$ & -37.422 & 144.094 & 2010 2014 & 29 & 25 & 33.10 & 0.82 \\
\hline BE-Bra & MF & 51.308 & 4.520 & 1997 2014 & 42 & 23 & 22.96 & 0.82 \\
\hline BE-Lon & $\mathrm{CRO}$ & 50.552 & 4.746 & 2004 2014 & 2.7 & 0.5 & 20.91 & 0.80 \\
\hline BE-Vie & $\mathrm{MF}$ & 50.305 & 5.998 & 1996 2014 & 40 & 35.2 & 22.66 & 0.83 \\
\hline BR-Sa1 & $\mathrm{EBF}$ & -2.857 & -54.959 & 2002 2011 & 57.8 & 41 & 15.52 & 0.87 \\
\hline BR-Sa3 & EBF & -3.018 & -54.971 & 2000 2004 & 64 & 27 & 8.05 & 0.94 \\
\hline CA-Gro & MF & 48.217 & -82.156 & 2003 2014 & 43.3 & 30 & 26.28 & 0.80 \\
\hline CA-Man & ENF & 55.880 & -98.481 & 1994 2008 & 30 & 10 & 20.02 & 0.85 \\
\hline CA-NS2 & ENF & 55.906 & -98.525 & 2001 2005 & 20 & 16 & 18.55 & 0.85 \\
\hline CA-NS3 & ENF & 55.912 & -98.382 & 2001 2005 & 10 & 7 & 22.53 & 0.82 \\
\hline
\end{tabular}




\begin{tabular}{|c|c|c|c|c|c|c|c|c|}
\hline $\begin{array}{c}\text { Site } \\
\text { name }\end{array}$ & IGBP & LAT & LONG & Year & $\begin{array}{c}\text { Measurement } \\
\text { height }(\mathrm{m})\end{array}$ & $\begin{array}{c}\text { Canopy } \\
\text { height }(\mathrm{m})\end{array}$ & $\begin{array}{l}\text { Energy } \\
\text { balance } \\
\text { residual } \\
\left(\mathrm{W} \mathrm{m}^{-2}\right)\end{array}$ & $\begin{array}{c}\text { Energy } \\
\text { balance } \\
\text { closure } \\
\text { ratio }\end{array}$ \\
\hline CA-NS5 & ENF & 55.863 & -98.485 & $2001 \sim 2005$ & 9 & 2 & 32.79 & 0.74 \\
\hline CA-Oas & DBF & 53.629 & -106.198 & 1996 2010 & 39 & 22 & 15.09 & 0.87 \\
\hline CA-Qfo & ENF & 49.693 & -74.342 & 2003 2010 & 24 & 13.8 & 26.57 & 0.80 \\
\hline CA-SF3 & $\mathrm{OSH}$ & 54.092 & -106.005 & 2001 2006 & 20 & 1 & 3.83 & 0.96 \\
\hline CA-TP1 & ENF & 42.661 & -80.560 & 2002 2014 & 3 & 2.8 & 24.44 & 0.81 \\
\hline CA-TP3 & ENF & 42.707 & -80.348 & 2002 2014 & 16 & 13.1 & 18.51 & 0.87 \\
\hline CA-TP4 & ENF & 42.710 & -80.357 & 2002 2014 & 28 & 21.8 & 36.15 & 0.78 \\
\hline CH-Cha & GRA & 47.210 & 8.410 & 2006 2012 & 2 & 0.5 & 1.57 & 0.99 \\
\hline CH-Dav & ENF & 46.815 & 9.856 & 1997 2011 & 35 & 18 & 42.06 & 0.70 \\
\hline CH-Fru & GRA & 47.116 & 8.538 & 2006 2012 & 2 & 0.5 & 7.38 & 0.94 \\
\hline CH-Oe1 & GRA & 47.286 & 7.732 & $2003 \sim 2008$ & 1.2 & 0.5 & 15.12 & 0.86 \\
\hline CZ-BK1 & ENF & 49.502 & 18.537 & 2004 2014 & 15 & 9.5 & 22.70 & 0.83 \\
\hline DE-Geb & CRO & 51.100 & 10.914 & 2001 2014 & 6 & 1 & 7.91 & 0.92 \\
\hline DE-Gri & GRA & 50.950 & 13.513 & 2004 2014 & 3 & 0.5 & 40.78 & 0.64 \\
\hline DE-Hai & DBF & 51.079 & 10.453 & 2000 2009 & 43.5 & 33 & 5.44 & 0.95 \\
\hline DE-Lnf & $\mathrm{DBF}$ & 51.328 & 10.368 & 2002 2012 & 44 & 34 & 5.35 & 0.95 \\
\hline DE-Obe & ENF & 50.787 & 13.721 & 2008 2014 & 42 & 13.5 & 23.18 & 0.81 \\
\hline DE-Tha & ENF & 50.962 & 13.565 & 1996 2014 & 42 & 30 & 16.66 & 0.87 \\
\hline DK-Sor & DBF & 55.486 & 11.645 & $2003 \sim 2013$ & 57 & 25 & -0.15 & 1.00 \\
\hline ES-LJu & OSH & 36.927 & -2.752 & 2004 2013 & 2.5 & 0.5 & 23.42 & 0.83 \\
\hline FI-Hyy & ENF & 61.847 & 24.295 & 2005 2014 & 67.2 & 15 & 9.61 & 0.91 \\
\hline FI-Sod & ENF & 67.362 & 26.639 & 2002 2014 & 23 & 12.7 & -8.71 & 1.09 \\
\hline
\end{tabular}




\begin{tabular}{|c|c|c|c|c|c|c|c|c|}
\hline $\begin{array}{l}\text { Site } \\
\text { name }\end{array}$ & IGBP & LAT & LONG & Year & $\begin{array}{l}\text { Measurement } \\
\text { height (m) }\end{array}$ & $\begin{array}{c}\text { Canopy } \\
\text { height }(\mathrm{m})\end{array}$ & $\begin{array}{l}\text { Energy } \\
\text { balance } \\
\text { residual } \\
\left(\mathrm{W} \mathrm{m}^{-2}\right)\end{array}$ & $\begin{array}{c}\text { Energy } \\
\text { balance } \\
\text { closure } \\
\text { ratio }\end{array}$ \\
\hline FR-Fon & $\overline{D B F}$ & 48.476 & 2.780 & $2005 \sim 2014$ & 35 & 25 & 10.96 & 0.91 \\
\hline FR-Gri & CRO & 48.844 & 1.952 & 2004 2013 & 2.8 & 2 & 8.49 & 0.92 \\
\hline FR-LBr & ENF & 44.717 & -0.769 & 1996 2008 & 25 & 18 & 16.20 & 0.88 \\
\hline FR-Pue & $\mathrm{EBF}$ & 43.741 & 3.596 & 2004 2013 & 11 & 6 & 38.20 & 0.74 \\
\hline GF-Guy & EBF & 5.279 & -52.925 & 2004 2014 & 52 & 32 & 6.35 & 0.96 \\
\hline IT-Col & DBF & 41.849 & 13.588 & 1996 2014 & 25.2 & 20.2 & 43.08 & 0.73 \\
\hline IT-Cpz & EBF & 41.705 & 12.376 & 1997 2008 & 15 & 12 & 21.30 & 0.87 \\
\hline IT-Lav & ENF & 45.956 & 11.281 & 2004 2014 & 28 & 28 & 0.72 & 1.00 \\
\hline IT-Noe & $\mathrm{CSH}$ & 40.606 & 8.151 & 2004 2014 & 2 & 1.75 & 8.75 & 0.95 \\
\hline IT-Ren & ENF & 46.587 & 11.434 & 2002 2013 & 41 & 31 & -27.06 & 1.19 \\
\hline IT-Ro1 & DBF & 42.408 & 11.930 & 2001 2008 & 18.6 & 17 & 24.76 & 0.83 \\
\hline IT-Ro2 & $\mathrm{DBF}$ & 42.390 & 11.921 & 2002 2011 & 18.6 & 17 & 40.27 & 0.75 \\
\hline IT-SRo & ENF & 43.728 & 10.284 & 2000 2007 & 23.5 & 18 & 44.46 & 0.76 \\
\hline IT-Tor & GRA & 45.844 & 7.578 & 2008 2014 & 2.5 & 0.5 & 2.82 & 0.98 \\
\hline MY-PSO & EBF & 2.973 & 102.306 & 2003 2009 & 52 & 35 & 2.74 & 0.98 \\
\hline NL-Hor & GRA & 52.240 & 5.071 & 2004 2011 & 4.3 & 0.5 & 15.34 & 0.87 \\
\hline NL-Loo & ENF & 52.167 & 5.744 & 1997 2014 & 52 & 15.9 & 26.51 & 0.81 \\
\hline RU-Cok & OSH & 70.829 & 147.494 & 2003 2012 & 4.7 & 0.3 & 17.03 & 0.80 \\
\hline RU-Fyo & ENF & 56.462 & 32.922 & 1999 2014 & 42.8 & 34.8 & 5.79 & 0.95 \\
\hline RU-Sam & GRA & 72.374 & 126.496 & 2002 2014 & 4 & 0.1 & 21.68 & 0.77 \\
\hline US-Atq & WET & 70.470 & -157.409 & 2004 2008 & 2.5 & 0.5 & 10.61 & 0.87 \\
\hline US-Blo & ENF & 38.895 & -120.633 & 1997 2007 & 8.5 & 4 & 10.35 & 0.94 \\
\hline
\end{tabular}




\begin{tabular}{|c|c|c|c|c|c|c|c|c|}
\hline $\begin{array}{c}\text { Site } \\
\text { name }\end{array}$ & IGBP & LAT & LONG & Year & $\begin{array}{l}\text { Measurement } \\
\text { height (m) }\end{array}$ & $\begin{array}{c}\text { Canopy } \\
\text { height }(\mathrm{m})\end{array}$ & $\begin{array}{l}\text { Energy } \\
\text { balance } \\
\text { residual } \\
\left(\mathrm{W} \mathrm{m}^{-2}\right)\end{array}$ & $\begin{array}{c}\text { Energy } \\
\text { balance } \\
\text { closure } \\
\text { ratio }\end{array}$ \\
\hline US-Cop & GRA & 38.090 & -109.390 & 2001 2007 & 1.85 & 0.1 & 33.39 & 0.71 \\
\hline US-GBT & ENF & 41.366 & -106.240 & 2001 2005 & 29 & 1 & 19.72 & 0.86 \\
\hline US-GLE & ENF & 41.367 & -106.240 & 2005 2014 & 22.65 & 18 & 21.21 & 0.85 \\
\hline US-Goo & GRA & 34.255 & -89.874 & 2002 2006 & 4 & 0.3 & 25.45 & 0.81 \\
\hline US-Ha1 & DBF & 42.538 & -72.172 & 1992 2012 & 30 & 26 & 16.84 & 0.87 \\
\hline US-IB2 & GRA & 41.841 & -88.241 & 2005 2011 & 3.76 & 0.5 & 31.23 & 0.77 \\
\hline US-Los & WET & 46.083 & -89.979 & 2000 2010 & 10.2 & 2 & 29.20 & 0.76 \\
\hline US-Me2 & ENF & 44.452 & -121.557 & 2002 2014 & 32 & 16 & 13.14 & 0.92 \\
\hline US-Me3 & ENF & 44.315 & -121.608 & 2004 2009 & 12 & 3.1 & 23.60 & 0.83 \\
\hline $\begin{array}{c}\text { US- } \\
\text { MMS }\end{array}$ & DBF & 39.323 & -86.413 & 2000 2014 & 46 & 27 & 35.60 & 0.76 \\
\hline US-Ne1 & $\mathrm{CRO}$ & 41.165 & -96.477 & 2002 2013 & 6.2 & 2 & 26.98 & 0.81 \\
\hline US-Ne2 & $\mathrm{CRO}$ & 41.165 & -96.470 & 2001 2013 & 6.2 & 2 & 22.19 & 0.84 \\
\hline US-Ne3 & $\mathrm{CRO}$ & 41.180 & -96.440 & 2001 2013 & 6.2 & 2 & 16.36 & 0.88 \\
\hline US-NR1 & ENF & 40.033 & -105.546 & 1999 2014 & 21.5 & 11.4 & 26.46 & 0.83 \\
\hline US-SRC & MF & 31.908 & -110.840 & 2008 2014 & 4.25 & 1.7 & 22.01 & 0.82 \\
\hline US-SRG & GRA & 31.789 & -110.828 & 2008 2014 & 8 & 2.5 & 13.18 & 0.89 \\
\hline US-SRM & WSA & 31.821 & -110.866 & 2004 2014 & 6.4 & 2.5 & 17.72 & 0.87 \\
\hline US-Syv & MF & 46.242 & -89.348 & 2002 2008 & 36 & 27 & 15.20 & 0.87 \\
\hline US-Ton & WSA & 38.432 & -120.966 & 2001 2014 & 23 & 9.41 & 29.51 & 0.81 \\
\hline US-UMB & DBF & 45.560 & -84.714 & 2000 2014 & 46 & 22 & 8.22 & 0.94 \\
\hline US-UMd & DBF & 45.563 & -84.698 & 2007 2014 & 34 & 22 & 10.36 & 0.92 \\
\hline
\end{tabular}




\begin{tabular}{ccccccccc}
\hline $\begin{array}{c}\text { Site } \\
\text { name }\end{array}$ & IGBP & LAT & LONG & Year & $\begin{array}{c}\text { Measurement } \\
\text { height }(\mathrm{m})\end{array}$ & $\begin{array}{c}\text { Canopy } \\
\text { height }(\mathrm{m})\end{array}$ & $\begin{array}{c}\text { Energy } \\
\text { balance } \\
\text { residual } \\
\left(\mathrm{W} \mathrm{m}^{-2}\right)\end{array}$ & $\begin{array}{c}\text { Energy } \\
\text { balance } \\
\text { closure } \\
\text { ratio }\end{array}$ \\
\hline US-Var & GRA & 38.413 & -120.951 & $2001 \sim 2014$ & 2 & 0.3 & 13.09 & 0.89 \\
US-WCr & DBF & 45.806 & -90.080 & $1999 \sim 2014$ & 29.6 & 24 & 22.80 & 0.82 \\
US-Whs & OSH & 31.744 & -110.052 & $2007 \sim 2014$ & 6 & 1 & 16.83 & 0.87 \\
US-Wkg & GRA & 31.737 & -109.942 & $2004 \sim 2014$ & 6.4 & 0.5 & 12.78 & 0.89 \\
ZA-Kru & SAV & -25.020 & 31.497 & $2000 \sim 2013$ & 16 & 10 & 4.06 & 0.97 \\
\hline
\end{tabular}


Table S2. The NSE values of the SGC (NSE $)$ and PGC functions (NSEB) for each site at different time scales. The subscript $\mathrm{H}$ and $\mathrm{B}$ correspond to the sigmoid function proposed in Han \& Tian (2018) and the polynomial function proposed in Brutsaert (2015), respectively.

\begin{tabular}{|c|c|c|c|c|c|c|c|c|}
\hline \multirow{2}{*}{ Site name } & \multicolumn{4}{|c|}{$\mathrm{NSE}_{\mathrm{H}}$} & \multicolumn{4}{|c|}{$\mathrm{NSE}_{\mathrm{B}}$} \\
\hline & Day & Week & Month & Year & Day & Week & Month & Year \\
\hline AT-Neu & 0.88 & 0.90 & 0.87 & -1.18 & 0.89 & 0.91 & 0.94 & 0.92 \\
\hline AU-ASM & 0.63 & 0.67 & 0.75 & 0.72 & 0.64 & 0.68 & 0.76 & 0.74 \\
\hline AU-Cpr & -0.26 & 0.45 & 0.60 & 0.47 & -0.75 & 0.43 & 0.59 & 0.54 \\
\hline AU-DaS & 0.78 & 0.83 & 0.87 & 0.81 & 0.76 & 0.82 & 0.87 & 0.80 \\
\hline AU-Dry & 0.84 & 0.88 & 0.90 & 0.90 & 0.83 & 0.88 & 0.90 & 0.91 \\
\hline AU-How & 0.67 & 0.65 & 0.56 & 0.33 & 0.65 & 0.64 & 0.57 & 0.38 \\
\hline AU-Stp & 0.80 & 0.85 & 0.95 & 0.84 & 0.82 & 0.78 & 0.90 & 0.60 \\
\hline AU-Tum & 0.71 & 0.87 & 0.85 & 0.81 & 0.68 & 0.86 & 0.85 & 0.84 \\
\hline AU-Wom & -0.37 & 0.53 & 0.48 & -0.48 & -0.32 & 0.31 & 0.42 & 0.28 \\
\hline BE-Bra & 0.46 & 0.48 & 0.64 & 0.36 & 0.43 & 0.46 & 0.64 & 0.47 \\
\hline BE-Lon & 0.61 & 0.52 & 0.48 & -0.68 & 0.52 & 0.34 & 0.28 & -0.52 \\
\hline BE-Vie & 0.34 & 0.54 & 0.59 & 0.51 & 0.32 & 0.50 & 0.51 & 0.45 \\
\hline BR-Sa1 & 0.93 & 0.97 & 0.95 & 0.82 & 0.89 & 0.94 & 0.89 & 0.71 \\
\hline BR-Sa3 & 0.85 & 0.92 & 0.89 & 0.80 & 0.81 & 0.90 & 0.86 & 0.75 \\
\hline CA-Gro & 0.30 & 0.71 & 0.80 & 0.86 & 0.36 & 0.68 & 0.77 & 0.88 \\
\hline CA-Man & -0.44 & 0.46 & 0.34 & 0.53 & -0.61 & 0.45 & 0.31 & 0.51 \\
\hline CA-NS2 & 0.29 & 0.44 & 0.37 & -0.19 & 0.18 & 0.39 & 0.33 & -0.24 \\
\hline CA-NS3 & 0.26 & 0.49 & 0.42 & -1.22 & 0.13 & 0.32 & 0.21 & -2.90 \\
\hline CA-NS5 & 0.51 & 0.76 & 0.86 & -0.57 & 0.28 & 0.67 & 0.86 & -0.65 \\
\hline CA-Oas & 0.66 & 0.70 & 0.68 & 0.47 & 0.65 & 0.74 & 0.74 & 0.68 \\
\hline CA-Qfo & -0.60 & 0.28 & 0.27 & 0.42 & -0.68 & 0.25 & 0.19 & 0.52 \\
\hline CA-SF3 & 0.79 & 0.85 & 0.74 & 0.72 & 0.68 & 0.78 & 0.59 & 0.64 \\
\hline CA-TP1 & 0.20 & 0.30 & 0.36 & 0.66 & 0.16 & 0.27 & 0.31 & 0.65 \\
\hline CA-TP3 & -0.03 & 0.12 & 0.03 & -1.62 & -0.08 & 0.07 & -0.01 & -1.74 \\
\hline CA-TP4 & 0.14 & 0.18 & 0.27 & -0.34 & 0.08 & 0.13 & 0.23 & -0.26 \\
\hline $\mathrm{CH}-\mathrm{Cha}$ & 0.89 & 0.86 & 0.84 & 0.47 & 0.88 & 0.86 & 0.90 & 0.95 \\
\hline CH-Dav & 0.35 & 0.48 & 0.68 & 0.31 & 0.06 & 0.20 & 0.59 & 0.90 \\
\hline CH-Fru & 0.92 & 0.88 & 0.77 & -0.42 & 0.90 & 0.87 & 0.88 & 0.88 \\
\hline $\mathrm{CH}-\mathrm{Oe} 1$ & 0.84 & 0.78 & 0.66 & -2.29 & 0.79 & 0.72 & 0.72 & -0.43 \\
\hline CZ-BK1 & 0.04 & 0.20 & 0.48 & 0.31 & -0.15 & 0.09 & 0.44 & 0.29 \\
\hline DE-Geb & 0.58 & 0.61 & 0.72 & 0.32 & 0.54 & 0.58 & 0.73 & 0.30 \\
\hline DE-Gri & 0.89 & 0.87 & 0.88 & 0.21 & 0.88 & 0.85 & 0.83 & 0.70 \\
\hline DE-Hai & 0.54 & 0.61 & 0.62 & 0.54 & 0.48 & 0.57 & 0.58 & 0.63 \\
\hline DE-Lnf & 0.62 & 0.75 & 0.75 & -1.19 & 0.57 & 0.71 & 0.71 & -0.49 \\
\hline
\end{tabular}




\begin{tabular}{|c|c|c|c|c|c|c|c|c|}
\hline DE-Obe & 0.59 & 0.46 & 0.68 & 0.55 & 0.55 & 0.35 & 0.66 & 0.50 \\
\hline DE-Tha & -0.05 & 0.44 & 0.48 & 0.51 & 0.03 & 0.34 & 0.40 & 0.44 \\
\hline DK-Sor & 0.48 & 0.58 & 0.66 & 0.08 & 0.47 & 0.59 & 0.67 & 0.45 \\
\hline ES-LJu & -0.53 & 0.04 & 0.12 & -0.66 & -0.95 & -0.24 & -0.28 & -1.41 \\
\hline FI-Hyy & 0.21 & -0.01 & 0.69 & 0.79 & 0.05 & -0.40 & 0.68 & 0.79 \\
\hline FI-Sod & 0.35 & 0.61 & 0.26 & 0.21 & 0.27 & 0.61 & 0.26 & 0.22 \\
\hline FR-Fon & 0.73 & 0.80 & 0.84 & 0.65 & 0.70 & 0.81 & 0.84 & 0.04 \\
\hline FR-Gri & 0.59 & 0.53 & 0.51 & 0.58 & 0.53 & 0.50 & 0.44 & 0.60 \\
\hline FR-LBr & -0.03 & 0.36 & 0.45 & 0.63 & 0.04 & 0.32 & 0.41 & 0.68 \\
\hline FR-Pue & -0.23 & 0.44 & 0.56 & 0.14 & -0.21 & 0.43 & 0.56 & 0.03 \\
\hline GF-Guy & 0.53 & 0.04 & -1.27 & 0.41 & 0.44 & -0.04 & -1.45 & -0.04 \\
\hline IT-Col & 0.49 & 0.81 & 0.88 & 0.80 & 0.40 & 0.79 & 0.88 & 0.81 \\
\hline IT-Cpz & -0.86 & -0.89 & -1.15 & -5.55 & -0.96 & -0.95 & -1.20 & -5.50 \\
\hline IT-Lav & -2.94 & -4.97 & -1.10 & -6.57 & -4.45 & -7.48 & -1.26 & -7.25 \\
\hline IT-Noe & -2.08 & -0.72 & -0.31 & 0.11 & -3.68 & -1.21 & -0.54 & 0.12 \\
\hline IT-Ren & -0.99 & 0.29 & 0.53 & 0.54 & -2.35 & 0.09 & 0.43 & 0.43 \\
\hline IT-Ro1 & 0.32 & 0.47 & 0.57 & 0.19 & 0.26 & 0.46 & 0.57 & 0.32 \\
\hline IT-Ro2 & 0.59 & 0.65 & 0.71 & 0.77 & 0.56 & 0.66 & 0.73 & 0.81 \\
\hline IT-SRo & -0.56 & -0.63 & -0.31 & 0.50 & -0.87 & -0.85 & -0.48 & 0.47 \\
\hline IT-Tor & 0.84 & 0.86 & 0.92 & 0.75 & 0.74 & 0.75 & 0.88 & 0.52 \\
\hline MY-PSO & -0.76 & -1.64 & -2.47 & 0.34 & -1.19 & -2.17 & -3.04 & 0.13 \\
\hline NL-Hor & 0.90 & 0.86 & 0.85 & -0.78 & 0.90 & 0.90 & 0.92 & 0.80 \\
\hline NL-Loo & 0.08 & 0.17 & 0.26 & 0.70 & -0.33 & -0.25 & -0.07 & 0.64 \\
\hline RU-Cok & 0.69 & 0.75 & 0.84 & 0.69 & 0.51 & 0.64 & 0.82 & 0.74 \\
\hline RU-Fyo & 0.37 & 0.67 & 0.72 & 0.76 & 0.36 & 0.66 & 0.70 & 0.77 \\
\hline RU-Sam & 0.82 & 0.84 & 0.82 & 0.89 & 0.66 & 0.80 & 0.74 & 0.77 \\
\hline US-Atq & 0.65 & -0.82 & 0.91 & 0.78 & 0.44 & -2.64 & 0.92 & 0.78 \\
\hline US-Blo & 0.50 & 0.68 & 0.76 & 0.22 & 0.46 & 0.67 & 0.75 & 0.23 \\
\hline US-Cop & -0.31 & -0.19 & -0.76 & -0.21 & -0.78 & -0.58 & -1.21 & -0.71 \\
\hline US-GBT & -0.60 & -1.04 & 0.83 & 0.36 & -1.37 & -2.44 & 0.81 & 0.40 \\
\hline US-GLE & -0.09 & 0.60 & 0.68 & 0.56 & -0.01 & 0.52 & 0.63 & 0.62 \\
\hline US-Goo & 0.81 & 0.73 & 0.61 & 0.51 & 0.76 & 0.67 & 0.40 & 0.47 \\
\hline US-Ha1 & 0.76 & 0.80 & 0.84 & 0.91 & 0.75 & 0.84 & 0.88 & 0.92 \\
\hline US-IB2 & 0.84 & 0.84 & 0.85 & -0.92 & 0.79 & 0.78 & 0.82 & -0.21 \\
\hline US-Los & 0.83 & 0.87 & 0.88 & 0.68 & 0.79 & 0.84 & 0.87 & 0.66 \\
\hline US-Me2 & -0.37 & 0.72 & 0.78 & 0.36 & -0.75 & 0.72 & 0.78 & 0.39 \\
\hline US-Me3 & 0.00 & 0.75 & 0.80 & 0.74 & -0.60 & 0.74 & 0.81 & 0.79 \\
\hline US-MMS & 0.80 & 0.90 & 0.91 & 0.34 & 0.79 & 0.90 & 0.93 & 0.45 \\
\hline US-Ne1 & 0.82 & 0.87 & 0.89 & 0.90 & 0.81 & 0.88 & 0.92 & 0.84 \\
\hline US-Ne2 & 0.80 & 0.86 & 0.85 & 0.85 & 0.79 & 0.85 & 0.86 & 0.80 \\
\hline US-Ne3 & 0.79 & 0.88 & 0.89 & 0.95 & 0.77 & 0.87 & 0.90 & 0.95 \\
\hline US-NR1 & 0.01 & -0.81 & 0.72 & 0.67 & -0.04 & -1.23 & 0.70 & 0.70 \\
\hline US-SRC & 0.66 & 0.75 & 0.76 & 0.78 & 0.63 & 0.74 & 0.76 & 0.78 \\
\hline US-SRG & 0.65 & 0.73 & 0.75 & 0.62 & 0.66 & 0.74 & 0.76 & 0.61 \\
\hline
\end{tabular}




\begin{tabular}{ccccccccc} 
US-SRM & 0.61 & 0.72 & 0.76 & 0.66 & 0.60 & 0.72 & 0.77 & 0.66 \\
US-Syv & 0.74 & 0.81 & 0.81 & 0.12 & 0.64 & 0.74 & 0.70 & 0.48 \\
US-Ton & 0.30 & 0.37 & 0.35 & 0.08 & 0.28 & 0.37 & 0.36 & 0.06 \\
US-UMB & 0.41 & 0.72 & 0.76 & 0.71 & 0.41 & 0.72 & 0.76 & 0.70 \\
US-UMd & 0.64 & 0.74 & 0.71 & 0.81 & 0.62 & 0.74 & 0.70 & 0.81 \\
US-Var & 0.49 & 0.63 & 0.73 & 0.83 & 0.16 & 0.37 & 0.41 & 0.74 \\
US-WCr & 0.79 & 0.84 & 0.87 & 0.70 & 0.76 & 0.85 & 0.86 & 0.54 \\
US-Whs & 0.74 & 0.84 & 0.88 & 0.14 & 0.72 & 0.84 & 0.90 & -0.02 \\
US-Wkg & 0.73 & 0.82 & 0.86 & 0.77 & 0.71 & 0.83 & 0.88 & 0.80 \\
ZA-Kru & 0.77 & 0.83 & 0.87 & 0.89 & 0.77 & 0.83 & 0.88 & 0.89 \\
\hline
\end{tabular}

Table S3. The evaluation merits (NSE, $\mathrm{R}^{2}$ and RMSE in $\mathrm{W} \mathrm{m}^{-2}$ ) of the two generalized complementary functions for each ecosystem types. The subscript $\mathrm{H}$ and B correspond to the sigmoid function proposed in Han \& Tian (2018) and the polynomial function proposed in Brutsaert (2015), respectively. The suffixes “_d”, “_w”, “_m”, “_y" represents the results at the daily, weekly, monthly and yearly, respectively.

\begin{tabular}{lcccccc}
\hline & $\mathrm{NSE}_{\mathrm{H}}$ & $\mathrm{R}_{\mathrm{H}}^{2}$ & $\mathrm{RMSE}_{\mathrm{H}}$ & $\mathrm{NSE}_{\mathrm{B}}$ & $\mathrm{R}^{2}{ }_{\mathrm{B}}$ & $\mathrm{RMSE}_{\mathrm{B}}$ \\
\hline CRO_d & 0.70 & 0.72 & 22.16 & 0.66 & 0.72 & 23.26 \\
CRO_w & 0.71 & 0.74 & 17.26 & 0.67 & 0.75 & 18.09 \\
CRO_m & 0.72 & 0.75 & 14.45 & 0.69 & 0.77 & 14.47 \\
CRO_y & 0.49 & 0.61 & 5.05 & 0.50 & 0.62 & 5.33 \\
CSH_d & -2.08 & 0.00 & 46.67 & -3.68 & 0.00 & 61.72 \\
CSH_w & -0.72 & 0.05 & 29.26 & -1.21 & 0.03 & 34.22 \\
CSH_m & -0.31 & 0.10 & 22.52 & -0.54 & 0.11 & 24.48 \\
CSH_y & 0.11 & 0.17 & 17.28 & 0.12 & 0.21 & 17.12 \\
DBF_d & 0.60 & 0.65 & 24.60 & 0.57 & 0.63 & 25.46 \\
DBF_w & 0.72 & 0.73 & 18.05 & 0.72 & 0.74 & 17.92 \\
DBF_m & 0.75 & 0.77 & 14.84 & 0.76 & 0.78 & 14.52 \\
DBF_y & 0.44 & 0.62 & 6.56 & 0.51 & 0.66 & 6.24 \\
EBF_d & 0.10 & 0.58 & 24.13 & 0.02 & 0.56 & 25.18 \\
EBF_w & 0.15 & 0.64 & 14.95 & 0.03 & 0.62 & 16.08 \\
EBF_m & -0.15 & 0.62 & 12.36 & -0.26 & 0.61 & 12.93 \\
EBF_y & -0.34 & 0.53 & 7.97 & -0.35 & 0.54 & 7.89 \\
ENF_d & -0.08 & 0.50 & 31.24 & -0.33 & 0.49 & 34.59 \\
ENF_w & 0.10 & 0.64 & 21.44 & -0.15 & 0.63 & 23.68 \\
ENF_m & 0.45 & 0.71 & 14.37 & 0.39 & 0.71 & 15.07
\end{tabular}




\begin{tabular}{lcccccc} 
ENF_y & 0.02 & 0.53 & 7.94 & -0.05 & 0.54 & 7.86 \\
GRA_d & 0.73 & 0.81 & 16.36 & 0.65 & 0.80 & 18.65 \\
GRA_w & 0.75 & 0.82 & 13.29 & 0.68 & 0.82 & 15.08 \\
GRA_m & 0.75 & 0.84 & 10.90 & 0.68 & 0.84 & 11.88 \\
GRA_y & 0.01 & 0.75 & 7.58 & 0.49 & 0.77 & 5.78 \\
MF_d & 0.50 & 0.64 & 21.78 & 0.48 & 0.63 & 22.45 \\
MF_w & 0.66 & 0.71 & 14.51 & 0.62 & 0.72 & 15.37 \\
MF_m & 0.72 & 0.75 & 11.42 & 0.68 & 0.77 & 12.32 \\
MF_y & 0.53 & 0.57 & 6.90 & 0.61 & 0.65 & 6.59 \\
OSH_d & 0.42 & 0.65 & 21.23 & 0.24 & 0.64 & 25.07 \\
OSH_w & 0.62 & 0.74 & 13.82 & 0.51 & 0.72 & 15.64 \\
OSH_m & 0.64 & 0.79 & 10.37 & 0.51 & 0.79 & 11.67 \\
OSH_y & 0.22 & 0.66 & 6.32 & -0.01 & 0.67 & 6.94 \\
SAV_d & 0.53 & 0.69 & 20.44 & 0.40 & 0.67 & 22.23 \\
SAV_w & 0.75 & 0.76 & 14.06 & 0.74 & 0.77 & 14.14 \\
SAV_m & 0.81 & 0.83 & 10.61 & 0.81 & 0.83 & 10.48 \\
SAV_y & 0.77 & 0.82 & 6.50 & 0.79 & 0.82 & 6.34 \\
WET_d & 0.74 & 0.83 & 16.24 & 0.62 & 0.80 & 19.52 \\
WET_w & 0.03 & 0.89 & 22.55 & -0.90 & 0.87 & 30.24 \\
WET_m & 0.89 & 0.92 & 7.77 & 0.90 & 0.93 & 7.59 \\
WET_y & 0.73 & 0.84 & 4.71 & 0.72 & 0.86 & 4.75 \\
WSA_d & 0.53 & 0.56 & 24.08 & 0.51 & 0.55 & 24.52 \\
WSA_w & 0.56 & 0.59 & 20.25 & 0.57 & 0.59 & 20.29 \\
WSA_m & 0.56 & 0.59 & 17.55 & 0.57 & 0.60 & 17.33 \\
WSA_y & 0.36 & 0.47 & 8.29 & 0.37 & 0.47 & 8.22 \\
\hline
\end{tabular}

Table S4. The evaluation merits (NSE, $\mathrm{R}^{2}$ and RMSE in $\mathrm{W} \mathrm{m}^{-2}$ ) of the two generalized complementary functions using the "Bowen ratio" (BR) closure correction method. The subscript H and B correspond to the SGC function proposed in Han \& Tian (2018) and the PGC function proposed in Brutsaert (2015), respectively.

\begin{tabular}{cccccc}
\hline & Day & Week & Month & Season & Year \\
\hline $\mathrm{NSE}_{\mathrm{H}}$ & 0.01 & 0.23 & 0.4 & 0.17 & -0.07 \\
$\mathrm{NSE}_{\mathrm{B}}$ & -0.28 & 0.03 & 0.27 & 0.11 & -0.23 \\
$\mathrm{R}_{\mathrm{H}}$ & 0.53 & 0.62 & 0.67 & 0.54 & 0.52 \\
$\mathrm{R}_{\mathrm{B}}$ & 0.52 & 0.61 & 0.68 & 0.55 & 0.52 \\
$\mathrm{RMSE}_{\mathrm{H}}$ & 26.62 & 18.9 & 14.56 & 11.3 & 7.88
\end{tabular}




\begin{tabular}{llllll} 
RMSE $_{B}$ & 29.77 & 20.59 & 15.26 & 11.3 & 8.03 \\
\hline
\end{tabular}


Figure S1. The estimated evaporation vs the observed site mean evaporation at seasonal scale based on the sigmoid function (a) and the polynomial function (b).

Each dot represents the site mean result $(\mathrm{N}=88$ in each panel). The regression equations and determination coefficients $\left(\mathrm{R}^{2}\right)$ were calculated by the site mean results of the $88 \mathrm{EC}$ sites. The subscript $\mathrm{H}$ and $\mathrm{B}$ correspond to the sigmoid function proposed in Han \& Tian (2018) and the polynomial function proposed in Brutsaert (2015), respectively.

(a)

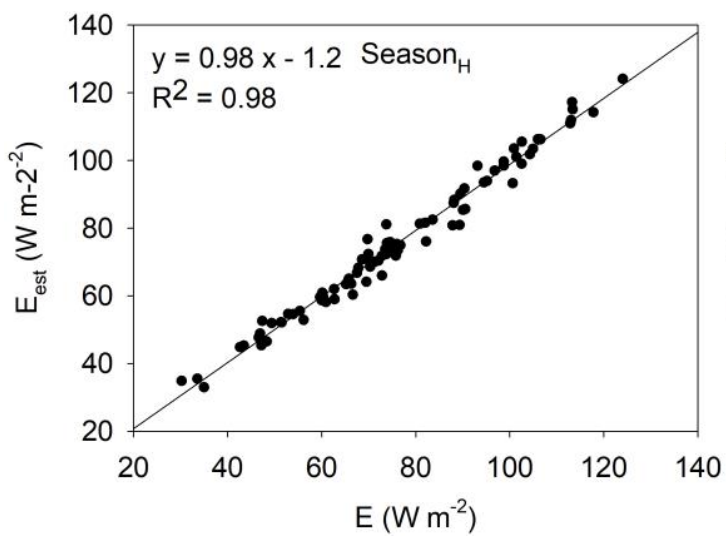

(b)

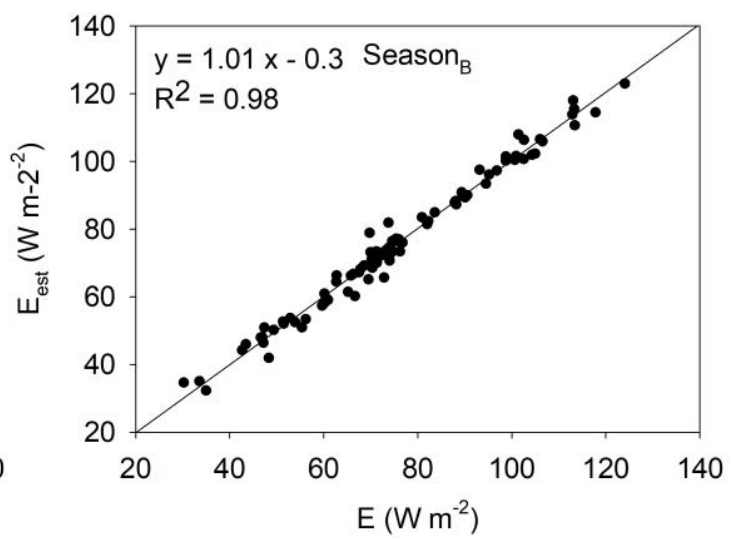


Reference list for dataset

\begin{tabular}{|c|c|c|c|c|}
\hline Site name & DOI & Creator & Title & $\begin{array}{c}\text { Date of last } \\
\text { access }\end{array}$ \\
\hline AT-Neu & 10.18140/FLX/1440121 & Georg Wohlfahrt & FLUXNET2015 AT-Neu Neustift & 2020.2 .15 \\
\hline AU-ASM & 10.18140/FLX/1440194 & James Cleverly & FLUXNET2015 AU-ASM Alice Springs & 2020.2 .15 \\
\hline AU-Cpr & 10.18140/FLX/1440195 & Wayne Meyer & FLUXNET2015 AU-Cpr Calperum & 2020.2 .15 \\
\hline AU-DaS & 10.18140/FLX/1440122 & Jason Beringer & FLUXNET2015 AU-DaS Daly River Cleared & 2020.2 .15 \\
\hline AU-Dry & 10.18140/FLX/1440197 & Jason Beringer & FLUXNET2015 AU-Dry Dry River & 2020.2 .15 \\
\hline AU-How & 10.18140/FLX/1440125 & Jason Beringer & FLUXNET2015 AU-How Howard Springs & 2020.2 .15 \\
\hline AU-Stp & 10.18140/FLX/1440204 & Jason Beringer & FLUXNET2015 AU-Stp Sturt Plains & 2020.2 .15 \\
\hline AU-Tum & 10.18140/FLX/1440126 & William Woodgate & FLUXNET2015 AU-Tum Tumbarumba & 2020.2 .15 \\
\hline AU-Wom & 10.18140/FLX/1440207 & Stefan Arndt & FLUXNET2015 AU-Wom Wombat & 2020.2 .15 \\
\hline BE-Bra & 10.18140/FLX/1440128 & Ivan Janssens & FLUXNET2015 BE-Bra Brasschaat & 2020.2 .15 \\
\hline BE-Lon & 10.18140/FLX/1440129 & Anne De Ligne & FLUXNET2015 BE-Lon Lonzee & 2020.2.15 \\
\hline BE-Vie & 10.18140/FLX/1440130 & Anne De Ligne & FLUXNET2015 BE-Vie Vielsalm & 2020.2 .15 \\
\hline BR-Sa1 & 10.18140/FLX/1440032 & Scott Saleska & FLUXNET2015 BR-Sa1 Santarem-Km67-Primary Forest & 2020.2 .15 \\
\hline $\mathrm{BR}-\mathrm{Sa} 3$ & 10.18140/FLX/1440033 & Mike Goulden & FLUXNET2015 BR-Sa3 Santarem-Km83-Logged Forest & 2020.2 .15 \\
\hline CA-Gro & 10.18140/FLX/1440034 & Harry McCaughey & $\begin{array}{l}\text { FLUXNET2015 CA-Gro Ontario - Groundhog River, Boreal } \\
\text { Mixedwood Forest }\end{array}$ & 2020.2.15 \\
\hline CA-Man & 10.18140/FLX/1440035 & Brian Amiro & $\begin{array}{l}\text { FLUXNET2015 CA-Man Manitoba - Northern Old Black } \\
\text { Spruce (former BOREAS Northern Study Area) }\end{array}$ & 2020.2.15 \\
\hline CA-NS2 & 10.18140/FLX/1440037 & Mike Goulden & FLUXNET2015 CA-NS2 UCI-1930 burn site & 2020.2 .15 \\
\hline CA-NS3 & 10.18140/FLX/1440038 & Mike Goulden & FLUXNET2015 CA-NS3 UCI-1964 burn site & 2020.2 .15 \\
\hline CA-NS5 & 10.18140/FLX/1440040 & Mike Goulden & FLUXNET2015 CA-NS5 UCI-1981 burn site & 2020.2 .15 \\
\hline
\end{tabular}




\begin{tabular}{|c|c|c|c|c|}
\hline Site name & DOI & Creator & Title & $\begin{array}{l}\text { Date of last } \\
\text { access }\end{array}$ \\
\hline CA-Oas & 10.18140/FLX/1440043 & T. Andrew Black & $\begin{array}{l}\text { FLUXNET2015 CA-Oas Saskatchewan - Western Boreal, } \\
\text { Mature Aspen }\end{array}$ & 2020.2 .15 \\
\hline CA-Qfo & 10.18140/FLX/1440045 & Hank A. Margolis & $\begin{array}{l}\text { FLUXNET2015 CA-Qfo Quebec - Eastern Boreal, Mature } \\
\text { Black Spruce }\end{array}$ & 2020.2 .15 \\
\hline CA-SF3 & 10.18140/FLX/1440048 & Brian Amiro & $\begin{array}{l}\text { FLUXNET2015 CA-SF3 Saskatchewan - Western Boreal, } \\
\text { forest burned in } 1998\end{array}$ & 2020.2 .15 \\
\hline CA-TP1 & 10.18140/FLX/1440050 & M. Altaf Arain & $\begin{array}{c}\text { FLUXNET2015 CA-TP1 Ontario - Turkey Point } 2002 \\
\text { Plantation White Pine }\end{array}$ & 2020.2 .15 \\
\hline CA-TP3 & 10.18140/FLX/1440052 & M. Altaf Arain & $\begin{array}{c}\text { FLUXNET2015 CA-TP3 Ontario - Turkey Point } 1974 \\
\text { Plantation White Pine }\end{array}$ & 2020.2 .15 \\
\hline CA-TP4 & 10.18140/FLX/1440053 & M. Altaf Arain & $\begin{array}{c}\text { FLUXNET2015 CA-TP4 Ontario - Turkey Point } 1939 \\
\text { Plantation White Pine }\end{array}$ & 2020.2 .15 \\
\hline $\mathrm{CH}-\mathrm{Cha}$ & 10.18140/FLX/1440131 & Lukas Hörtnagl & FLUXNET2015 CH-Cha Chamau & 2020.2 .15 \\
\hline CH-Dav & 10.18140/FLX/1440132 & Lukas Hörtnagl & FLUXNET2015 CH-Dav Davos & 2020.2 .15 \\
\hline CH-Fru & 10.18140/FLX/1440133 & Lukas Hörtnagl & FLUXNET2015 CH-Fru Früebüel & 2020.2 .15 \\
\hline $\mathrm{CH}-\mathrm{Oe} 1$ & 10.18140/FLX/1440135 & Christoph Ammann & FLUXNET2015 CH-Oe1 Oensingen grassland & 2020.2 .15 \\
\hline CZ-BK1 & 10.18140/FLX/1440143 & Ladislav Sigut & FLUXNET2015 CZ-BK1 Bily Kriz forest & 2020.2 .15 \\
\hline DE-Geb & 10.18140/FLX/1440146 & Christian Brümmer & FLUXNET2015 DE-Geb Gebesee & 2020.2 .15 \\
\hline DE-Gri & 10.18140/FLX/1440147 & Christian Bernhofer & FLUXNET2015 DE-Gri Grillenburg & 2020.2 .15 \\
\hline DE-Hai & 10.18140/FLX/1440148 & Alexander Knohl & FLUXNET2015 DE-Hai Hainich & 2020.2 .15 \\
\hline DE-Lnf & 10.18140/FLX/1440150 & Alexander Knohl & FLUXNET2015 DE-Lnf Leinefelde & 2020.2 .15 \\
\hline DE-Obe & 10.18140/FLX/1440151 & Christian Bernhofer & FLUXNET2015 DE-Obe Oberbärenburg & 2020.2 .15 \\
\hline DE-Tha & 10.18140/FLX/1440152 & Christian Bernhofer & FLUXNET2015 DE-Tha Tharandt & 2020.2 .15 \\
\hline DK-Sor & 10.18140/FLX/1440155 & Andreas Ibrom & FLUXNET2015 DK-Sor Soroe & 2020.2 .15 \\
\hline
\end{tabular}




\begin{tabular}{|c|c|c|c|c|}
\hline Site name & DOI & Creator & Title & $\begin{array}{c}\text { Date of last } \\
\text { access }\end{array}$ \\
\hline ES-LJu & 10.18140/FLX/1440157 & $\begin{array}{c}\text { Erique Pérez Sánchez } \\
\text { Cañete }\end{array}$ & FLUXNET2015 ES-LJu Llano de los Juanes & 2020.2 .15 \\
\hline FI-Hyy & 10.18140/FLX/1440158 & Ivan Mammarella & FLUXNET2015 FI-Hyy Hyytiala & 2020.2 .15 \\
\hline FI-Sod & 10.18140/FLX/1440160 & Mika Aurela & FLUXNET2015 FI-Sod Sodankyla & 2020.2.15 \\
\hline FR-Fon & 10.18140/FLX/1440161 & Daniel Berveiller & FLUXNET2015 FR-Fon Fontainebleau-Barbeau & 2020.2.15 \\
\hline FR-Gri & 10.18140/FLX/1440162 & Pauline Buysse & FLUXNET2015 FR-Gri Grignon & 2020.2 .15 \\
\hline FR-LBr & 10.18140/FLX/1440163 & Paul Berbigier & FLUXNET2015 FR-LBr Le Bray & 2020.2 .15 \\
\hline FR-Pue & 10.18140/FLX/1440164 & Jean-Marc Ourcival & FLUXNET2015 FR-Pue Puechabon & 2020.2 .15 \\
\hline GF-Guy & 10.18140/FLX/1440165 & Damien BONAL & FLUXNET2015 GF-Guy Guyaflux (French Guiana) & 2020.2 .15 \\
\hline IT-Col & 10.18140/FLX/1440167 & Giorgio Matteucci & FLUXNET2015 IT-Col Collelongo & 2020.2 .15 \\
\hline IT-Cpz & 10.18140/FLX/1440168 & Riccardo Valentini & FLUXNET2015 IT-Cpz Castelporziano & 2020.2 .15 \\
\hline IT-Lav & 10.18140/FLX/1440169 & Damiano Gianelle & FLUXNET2015 IT-Lav Lavarone & 2020.2 .15 \\
\hline IT-Noe & 10.18140/FLX/1440171 & Donatella Spano & FLUXNET2015 IT-Noe Arca di Noe - Le Prigionette & 2020.2 .15 \\
\hline IT-Ren & 10.18140/FLX/1440173 & Leonardo Montagnani & FLUXNET2015 IT-Ren Renon & 2020.2 .15 \\
\hline IT-Ro1 & 10.18140/FLX/1440174 & Riccardo Valentini & FLUXNET2015 IT-Ro1 Roccarespampani 1 & 2020.2 .15 \\
\hline IT-Ro2 & 10.18140/FLX/1440175 & Dario Papale & FLUXNET2015 IT-Ro2 Roccarespampani 2 & 2020.2 .15 \\
\hline IT-SRo & 10.18140/FLX/1440176 & Carsten Gruening & FLUXNET2015 IT-SRo San Rossore & 2020.2 .15 \\
\hline IT-Tor & 10.18140/FLX/1440237 & Edoardo Cremonese & FLUXNET2015 IT-Tor Torgnon & 2020.2 .15 \\
\hline MY-PSO & 10.18140/FLX/1440240 & Yoshiko Kosugi & FLUXNET2015 MY-PSO Pasoh Forest Reserve (PSO) & 2020.2 .15 \\
\hline NL-Hor & 10.18140/FLX/1440177 & Han Dolman & FLUXNET2015 NL-Hor Horstermeer & 2020.2 .15 \\
\hline NL-Loo & 10.18140/FLX/1440178 & Eddy Moors & FLUXNET2015 NL-Loo Loobos & 2020.2 .15 \\
\hline RU-Cok & 10.18140/FLX/1440182 & Han Dolman & FLUXNET2015 RU-Cok Chokurdakh & 2020.2 .15 \\
\hline RU-Fyo & 10.18140/FLX/1440183 & Andrej Varlagin & FLUXNET2015 RU-Fyo Fyodorovskoye & 2020.2 .15 \\
\hline RU-Sam & 10.18140/FLX/1440185 & Lars Kutzbach & FLUXNET2015 RU-Sam Samoylov & 2020.2 .15 \\
\hline
\end{tabular}




\begin{tabular}{|c|c|c|c|c|}
\hline Site name & DOI & Creator & Title & $\begin{array}{l}\text { Date of last } \\
\text { access }\end{array}$ \\
\hline US-Atq & 10.18140/FLX/1440067 & Donatella Zona & FLUXNET2015 US-Atq Atqasuk & 2020.2 .15 \\
\hline US-Blo & 10.18140/FLX/1440068 & Allen Goldstein & FLUXNET2015 US-Blo Blodgett Forest & 2020.2.15 \\
\hline US-Cop & 10.18140/FLX/1440100 & David Bowling & FLUXNET2015 US-Cop Corral Pocket & 2020.2.15 \\
\hline US-GBT & 10.18140/FLX/1440118 & Bill Massman & FLUXNET2015 US-GBT GLEES Brooklyn Tower & 2020.2 .15 \\
\hline US-GLE & 10.18140/FLX/1440069 & Bill Massman & FLUXNET2015 US-GLE GLEES & 2020.2 .15 \\
\hline US-Goo & 10.18140/FLX/1440070 & Tilden Meyers & FLUXNET2015 US-Goo Goodwin Creek & 2020.2 .15 \\
\hline US-Ha1 & 10.18140/FLX/1440071 & J. William Munger & $\begin{array}{l}\text { FLUXNET2015 US-Ha1 Harvard Forest EMS Tower } \\
\text { (HFR1) }\end{array}$ & 2020.2.15 \\
\hline US-IB2 & 10.18140/FLX/1440072 & Roser Matamala & $\begin{array}{l}\text { FLUXNET2015 US-IB2 Fermi National Accelerator } \\
\text { Laboratory- Batavia (Prairie site) }\end{array}$ & 2020.2.15 \\
\hline US-Los & 10.18140/FLX/1440076 & Ankur Desai & FLUXNET2015 US-Los Lost Creek & 2020.2 .15 \\
\hline US-Me2 & 10.18140/FLX/1440079 & Ankur Desai & FLUXNET2015 US-Los Lost Creek & 2020.2 .15 \\
\hline US-Me3 & 10.18140/FLX/1440080 & Bev Law & FLUXNET2015 US-Me3 Metolius-second young aged pine & 2020.2 .15 \\
\hline US-MMS & 10.18140/FLX/1440083 & Kim Novick & FLUXNET2015 US-MMS Morgan Monroe State Forest & 2020.2 .15 \\
\hline US-Ne1 & 10.18140/FLX/1440084 & Andy Suyker & $\begin{array}{l}\text { FLUXNET2015 US-Ne1 Mead - irrigated continuous maize } \\
\text { site }\end{array}$ & 2020.2.15 \\
\hline US-Ne2 & 10.18140/FLX/1440085 & Andy Suyker & $\begin{array}{l}\text { FLUXNET2015 US-Ne2 Mead - irrigated maize-soybean } \\
\text { rotation site }\end{array}$ & 2020.2.15 \\
\hline US-Ne3 & 10.18140/FLX/1440086 & Andy Suyker & $\begin{array}{l}\text { FLUXNET2015 US-Ne3 Mead - rainfed maize-soybean } \\
\text { rotation site }\end{array}$ & 2020.2.15 \\
\hline US-NR1 & 10.18140/FLX/1440087 & Peter D. Blanken & $\begin{array}{l}\text { FLUXNET2015 US-NR1 Niwot Ridge Forest (LTER } \\
\text { NWT1) }\end{array}$ & 2020.2 .15 \\
\hline US-SRC & 10.18140/FLX/1440098 & Shirley Kurc & FLUXNET2015 US-SRC Santa Rita Creosote & 2020.2 .15 \\
\hline US-SRG & 10.18140/FLX/1440114 & Russell Scott & FLUXNET2015 US-SRG Santa Rita Grassland & 2020.2 .15 \\
\hline
\end{tabular}




\begin{tabular}{ccccc}
\hline Site name & DOI & Creator & Title & $\begin{array}{c}\text { Date of last } \\
\text { access }\end{array}$ \\
\hline US-SRM & $10.18140 /$ FLX/1440090 & Russell Scott & FLUXNET2015 US-SRM Santa Rita Mesquite & 2020.2 .15 \\
US-Syv & $10.18140 /$ FLX/1440091 & Ankur Desai & FLUXNET2015 US-Syv Sylvania Wilderness Area & 2020.2 .15 \\
US-Ton & $10.18140 /$ FLX/1440092 & Siyan Ma & FLUXNET2015 US-Ton Tonzi Ranch & 2020.2 .15 \\
US-UMB & $10.18140 /$ FLX/1440093 & Christopher Gough & FLUXNET2015 US-UMB Univ. of Mich. Biological Station & 2020.2 .15 \\
US-UMd & $10.18140 /$ FLX/1440101 & Christopher Gough & FLUXNET2015 US-UMd UMBS Disturbance & 2020.2 .15 \\
US-Var & $10.18140 /$ FLX/1440094 & Siyan Ma & FLUXNET2015 US-Var Vaira Ranch- Ione & 2020.2 .15 \\
US-WCr & $10.18140 /$ FLX/1440095 & Ankur Desai & FLUXNET2015 US-WCr Willow Creek & 2020.2 .15 \\
US-Whs & $10.18140 /$ FLX/1440097 & Russ Scott & FLUXNET2015 US-Whs Walnut Gulch Lucky Hills Shrub & 2020.2 .15 \\
US-Wkg & $10.18140 /$ FLX/1440096 & Russell Scott & FLUXNET2015 US-Wkg Walnut Gulch Kendall Grasslands & 2020.2 .15 \\
ZA-Kru & $10.18140 /$ FLX/1440188 & Bob Scholes & FLUXNET2015 ZA-Kru Skukuza & 2020.2 .15 \\
\hline
\end{tabular}

